\title{
SOCIOLOGÍA DEL TURISMO: EL SISTEMA TURÍSTICO FRENTE AL CAMBIO CLIMÁTICO
}

\section{SOCIOLOGY OF TOURISM: TOURIST SYSTEM ADDRESSING CLIMATE CHANGE}

Recibido/Received: 05/06/2010

Aceptado/Accepted: 09/09/2010

\section{RESUMEN}

Es evidente, hoy en día, que estamos ante una situación de cambio climático. Las temperaturas son extremas, el nivel del agua de los mares se incrementa, la capa de ozono desaparece, etc. No entramos a debatir las causas del proceso. Lo que nos interesa es analizar cómo esos cambios afectan al mapa turístico, es decir, queremos argumentar cómo el cambio climático afecta a los movimientos turísticos y establece nuevos destinos en relación a sus necesidades y expectativas viajeras. La subida de las temperaturas está haciendo que algunas zonas que hasta ahora eran netamente turísticas pierdan un buen margen de competitividad en favor de otras que estaban en las últimas posiciones. Pero también tenemos que tener en cuenta que si un eslabón de la cadena del sistema turístico se ve alterado, los demás también sufrirán alteraciones. En definitiva, en este artículo se analizarán los cambios a los que se verá sometido el turismo por la metamorfosis climática.

\section{PALABRAS CLAVE}

Necesidad, expectativa, destino turístico, transporte, viaje, sostenibilidad.

\section{SUMARIO}

1. Introducción. 2. El clima como uno de los factores clave del sistema turístico. 3. Cambio climático vs cambio turístico. 4. Conclusiones. Bibliografía.

\begin{abstract}
It is clear that today we face a situation of climate change. Temperatures are extreme, the water level of the sea increases, the ozone layer disappears, and so on. We do not care about the causes of this change. What interests us is to analyze how these changes affect the tourist map, that is, we argue how climate change affects tourism flows and sets new targets in relation to their needs and expectations of travelers. The rising temperatures are causing some areas that were previously lost a good tourist purely competitive edge for others who were in the final positions. But we must also bear in mind that if one link in the chain of the tourism system is altered, the others also suffer alterations. In short, this article will analyze the changes that will be placed under the tourism climate metamorphosis.
\end{abstract}

\section{KEYWORDS}

Needs, expectations, tourist, transportation, travel, sustainability. 


\section{CONTENTS}

1. Introduction. 2. The weather as one of the key system tourist. 3. Climate change vs tourism change. 4. Conclusions. References.

\section{INTRODUCCIÓN.}

Hoy, el turismo es un fenómeno social de primer nivel, y lo es porque mueve una cantidad de personas y de recursos absolutamente imponente. Por ello se nos hace necesario su análisis desde el punto de vista sociológico sobre todo en cuanto que es un indicador de desarrollo en muchas sociedades y supone una realidad dinámica que está sujeta a fluctuaciones permanentes. En este sentido queremos estudiar las transformaciones que en un futuro no muy lejano se podrían producir en el sistema turístico por las influencias de otro fenómeno que está aconteciendo con potencia en todo el mundo y que podría afectar al avance de las sociedades inmersas en los circuitos turísticos: el del cambio climático. Este hecho está muy ligado a la cuestión turística sin poder ser de otro modo, pues qué duda cabe que el clima es un elemento fundamental para que se establezcan los distintos flujos viajeros de unos lugares a otros.

Lo cierto es que la gran mayoría de los estudios que se han hecho respecto al cambio climático indican que éste es una realidad. Podemos decir que hay dos líneas abiertas para el debate: una, que es la menos secundada, afirma que las transformaciones que se están produciendo en el medio ambiente y en el clima están relacionadas con una metamorfosis cíclica natural que se da en nuestro planeta con el transcurso de ciertos períodos de tiempo, y que permanentemente el planeta Tierra ha sufrido innumerables cambios en las temperaturas que han incidido en la posterior conformación de unos ecosistemas y la desaparición de otros. Por otro lado existe otra línea de pensamiento, mucho más extendida y popular, que considera que los motivos del cambio climático y todas sus repercusiones están estrechamente relacionados con las malas e insostenibles actuaciones del ser humano que precipitan los cambios de una manera que no se había dado antes.

A nosotros no nos interesa demasiado, aunque sí algo, ese debate que insta a responsabilizar del cambio climático a la propia naturaleza o a la mano del hombre. Desde un punto de vista ecuánime posiblemente lo más certero sea no desdeñar ninguna de las dos posturas ya que existen estudios que confirman la influencia de ambas realidades en la aparición de dichos cambios. De hecho, aunque sea cierto que exista una permuta cíclica temporal en el clima por motivos naturales, no es menos cierto que la acción humana está provocando que esas alteraciones se aceleren y sus consecuencias también por lo que la inmediatez del cambio es lo que provoca que no se puedan determinar los impactos en su justa medida. Si a eso le añadimos que los efectos del cambio climático derivados de la acción humana están vinculados al desarrollo tecnológico e industrial más reciente de las sociedades avanzadas tenemos un cóctel explosivo que hace difícil adoptar alternativas reales para conciliar un medio ambiente sostenible con un desarrollo creciente e irrenunciable de las sociedades.

Por lo tanto, e independientemente de ambas posturas, lo que nos interesa es analizar qué repercusiones va a ocasionar el cambio climático en el sistema turístico, pues el elemento destino con sus recursos naturales y biológicos son pieza clave en la relación con el 
resto de los elementos del sistema. Las mutaciones climáticas traerán consigo variaciones en las motivaciones viajeras y los flujos turísticos estarán enfocados, si sigue la tendencia actual, hacia zonas más septentrionales del mundo. Los transportistas también tendrán que modificar rutas y quizá frecuencias, pues la estrecha relación entre la contaminación que provocan los transportes (sobre todo el aéreo) y el cambio climático cada vez cobra más fuerza, lo cual implica limitaciones en el tráfico de aeronaves, automóviles, barcos y demás medios para el traslado de personas y mercancías así como el desarrollo de tecnologías alternativas más limpias que reduzcan o anulen los impactos medioambientales.

\section{EL CLIMA COMO UNO DE LOS FACTORES CLAVE DEL SISTEMA TURÍSTICO}

La teoría de los sistemas está claramente aceptada en el ámbito del turismo. Ésta teoría se aplica para declarar que el turismo se constituye bajo un sistema que integra una serie de elementos que están relacionados e interconectados de manera tal, que si uno de los elementos del sistema se altera, tendrá repercusiones en el resto de los elementos de dicho conjunto. En el sistema turístico podemos diferenciar cinco tipos de elementos, a saber: los destinos; los consumidores; los transportistas; las agencias de viajes y las tecnologías (Albert, 2008:189). Los destinos son los distintos lugares geográficos que se establecen a lo largo y ancho del mundo y representan el objeto espacial absolutamente irrenunciable para que el fenómeno turístico sea una realidad. Tal y como dice Isabel Albert (2008), para poder identificar hoy un destino turístico es imprescindible que éste "cuente con cuatro tipos de recursos: recursos naturales, recursos culturales, infraestructura general y servicios turísticos. Cada uno de ellos incluye variados integrantes, que deben someterse a unas normas de autenticidad y calidad medioambiental y cultural, de acuerdo con las exigencias de la demanda actual". Efectivamente uno de los recursos de los destinos es el natural y dentro de él encontramos un componente que casi con toda seguridad se podría decir que es el primordial para que se produzca el turismo.

Decimos que el clima es, de inicio, fundamental porque en la actualidad incide claramente en la toma de decisión del consumidor turístico, no sólo como un hecho en el que se pretende la búsqueda de zonas climatológicamente placenteras, sino también en la forma que nos dice que el turismo está muy identificado con patrones de calidad en general y calidad ambiental en particular, lo cual está en estrecha relación con los sucesos climáticos. Efectivamente el clima es un factor motivacional de primera magnitud. Las actividades turísticas están en clara dependencia del clima que haga o vaya a hacer en los lugares de destino y entre los elementos climatológicos destacan, con respecto al turismo, la temperatura, la nieve, la lluvia, las nubes, el sol y el viento.

Lógicamente el clima no sólo hay que asumirlo desde un punto de vista aséptico, meramente natural, sino que en relación con el turismo lo tenemos que analizar socialmente para poder entenderlo. Por ejemplo la nieve podría ser un factor negativo para los desplazamientos de turistas que tuviesen la necesidad de temperaturas agradables en los destinos, pero al mismo tiempo puede ser un factor muy positivo para una demanda que tenga como objetivo fundamental el pasar una buena parte de su tiempo libre esquiando. $\mathrm{O}$ más esclarecedor aún, a nadie le resulta extraño en nuestra cotidianidad que una gran cantidad de personas busquen destinos con una climatología favorable buscando una coloración de la piel que está ligada, entre otras cuestiones, a los cánones de belleza 
socialmente establecidos y que nada tienen que ver con los modelos de belleza del siglo XIX que estaban relacionados con pieles lechosas, pálidas. El sol y playa no tendría las repercusiones actuales si los modelos sociales no se hubieran transformado.

Lo cierto es que debido a la diversificación y complejidad actual de la demanda el factor clima es muy importante para las multiformes necesidades de la misma ya sean éstas como recursos básicos o complementarios. Las condiciones climáticas presentadas en un momento dado pueden determinar la realización o no de diferentes actividades que van desde las ya mencionadas como sol y playa y deportes de invierno hasta prácticas como el golf, el senderismo o la pesca. Queda claro, por lo tanto, que el recurso natural "clima" es un factor clave en el desarrollo del turismo mundial y lo es, fundamentalmente, porque incide en bastantes aspectos de la actividad turística que podemos resumir en el decálogo ideado por Belén Gómez Martín (1999) donde expone sobre la relación clima-turismo lo siguiente:

- El clima actúa de cincel modelador de entornos ambientales variados que serán elegidos por los turistas dependiendo de lo que vayan buscando. Así, en las zonas más húmedas habrá más vegetación que en las más áridas y la fauna tendrá también distintas características en unos y otros enclaves.

- El clima tiene mucho que ver con la estacionalidad de la actividad turística y todo lo que ello conlleva. Muchas actividades están en dependencia del período estacional en que se esté. Por ejemplo, las zonas del litoral mediterráneo tienen unos flujos turísticos importantes en la temporada de verano y la riqueza de la zona está en gran parte supeditada al desarrollo de las actividades turísticas. "Estaciones largas permiten un mejor aprovechamiento de las infraestructuras y de los equipamientos $\mathrm{y}$, consecuentemente, permiten una mejor rentabilización del capital invertido. [...] Una mala adaptación de la actividad turística a las condiciones climatológicas o una escasa diversificación de las actividades en función de las diferentes condiciones registradas a lo largo del año potencian el problema de la estacionalidad" (Gómez, 1999: 26)

- El tipo de clima que se dé en un entorno y en un tiempo determinado tiene efectos en la actividad turística y en su planificación y más aún si esa actividad se realiza a la intemperie. Qué duda cabe que intentar contemplar una puesta de sol un día nublado puede dar al traste con las expectativas de un turista, o si éste quiere salir a navegar y hay una fuerte tormenta posiblemente desista de realizar tal ejercicio.

- El buen clima es ofrecido por las empresas turísticas utilizándolo como un efecto llamada hacia el cliente. El sector turístico tiene muy presente que por regla general el turista busca en sus desplazamientos destinos con condiciones meteorológicas favorables, incluso en el turismo de nieve el cliente espera disfrutar de jornadas de esquí bajo un apacible día soleado y no sobre una intempestiva tormenta blanca. Esto es debido al influjo de la cultura del sol, extendida muy uniformemente por las sociedades desarrolladas que consideran positivamente los amplios beneficios para la salud y la belleza que tiene el exponerse a este astro del que el planeta Tierra disfruta.

- Existe una incompatibilidad entre el turismo y los fenómenos climatológicos radicales adversos que son susceptibles de desembocar en catástrofes naturales. Para que el fenómeno turístico se establezca se necesitan unos requisitos mínimos de 
seguridad. Es un condicionamiento psicológico primario que nos dice que si no tenemos los niveles elementales de seguridad satisfechos no podemos pasar a otro estadio superior de necesidades. Un huracán, un tsunami, un cataclismo natural de cualquier índole genera inseguridad y miedo. Las zonas con mayor riesgo de catástrofes naturales requieren de una mayor planificación de gestión, de tiempo, de recursos, de prevención, de infraestructura adaptada a esas eventualidades extremas. Un lugar que ha sufrido una catástrofe natural puede tardar en recuperarse por la desconfianza que se le presenta al turista. Si la catástrofe se reitera puede suponer incluso un cese permanente de la actividad turística aunque dichos sucesos no hayan supuesto daños límite, pero la sensación y percepción del riesgo potencial en el turista queda instalado de una manera arraigada.

- El clima supedita el tipo de construcciones e infraestructuras de un destino. Las edificaciones que se establezcan en un lugar están en clara consonancia con su meteorología tipo, la cual deben tener en cuenta. En lugares con fuerte y permanente viento se alzan edificios con perfiles redondeados que faciliten la canalización equilibrada de ese exceso de energía eólica. Incluso la decisión en los colores de las fachadas o el material utilizado para las mismas inciden en el tipo de clima que convencionalmente se dé en un determinado lugar. Así muchos sitios donde el sol es una norma casi diaria utilizan colores claros o el blanco para repeler los rayos y mantener de alguna manera unas temperaturas no demasiado extremas en los alojamientos. Es curioso ver cómo algunas construcciones netamente turísticas como pueden ser complejos hoteleros de gran magnitud toman "muy en cuenta la importancia fundamental que en la moderación de determinados elementos atmosféricos tiene el empleo de arbolado, setos y vegetación en general: "desde la antigüedad se han usado las filas de los árboles, por ejemplo, como cortavientos de excepcional eficacia y se ha utilizado, en climas cálidos y secos, la jardinería, tanto por su valor ornamental como por sus efectos purificadores y refrescantes de los lugares habitables" (Gómez, 1999: 29)

- Los transportes y comunicaciones tienen un importante vínculo con los aspectos del clima de cara a su buen o mal funcionamiento. El turismo necesita de los transportes como medio de desplazamiento hacia las distintas zonas geográficas del mundo y éstos tienen en ocasiones incidencias negativas por no haber sido suficientemente valorado el factor meteorológico, sobre todo en lo que tiene que ver con el transporte terrestre (carreteras que muestran el desgaste provocado por las inclemencias meteorológicas, vías ferroviarias sin alternativas reales ante el colapso que pueda infundir una gran tormenta, etc.).

- El clima repercute en las sensaciones gratas y de disfrute del turista así como en las sensaciones desagradables y negativas. Dependiendo del clima que haga, éste nos va a marcar diferentes sensaciones. El tiempo que haga nos puede motivar sentimientos de alegría, tristeza, hastío, tranquilidad, euforia y otras muchas emociones más. El consumidor turístico va a tender hacia climas que le generen sensaciones psicológicas positivas y de disfrute.

- El clima va a tener repercusiones en la salud del turista y en las percepciones que éste tenga con respecto a conceptos como calidad y bienestar. Los ambientes meteorológicos favorables ayudan a nuestro organismo a mantenerse en buenas condiciones. Estos efectos positivos para nuestra salud se hacen más patentes, si 
cabe, conforme la edad va siendo más avanzada ya que los riesgos de sufrir algún problema de salud relacionado con el clima son mayores. Así se puede asegurar la incidencia que adquieren "los casos de infarto de miocardio que siguen a variaciones bruscas y pronunciadas del poder refrigerante del aire o a irrupciones de vientos violentos; los casos de accidentes vasculares cerebrales asociados en verano a un calor intenso y húmedo o a vientos muy cargados de vapor de agua; o los accidentes embólicos y las insuficiencias miocárdicas ligados a descensos bruscos de los valores barométricos" (Gómez, 1999: 31).

- De la misma manera el clima es un factor de medición de la calidad y de la satisfacción del consumidor turístico. Las variaciones que se establecen en los destinos por parte de los turistas viene dado, en gran medida, por los cambios meteorológicos que puedan aparecer en los diferentes lugares. El grado de satisfacción del turista, según una gran cantidad de estudios, se corresponde en un porcentaje superior al $80 \%$ a las buenas condiciones climatológicas presentadas en un lugar. El motivo "sol" tiene mucho gancho entre los turistas.

Acabamos de ver en unos cuantos puntos la gran relevancia que tiene el clima a la hora de configurar socialmente el turismo. Pero claro, el clima de un lugar determinado, a pesar de sufrir modificaciones a lo largo de un año, no deja de tener unas pautas más o menos firmes, estables, que hasta ahora nos permitían predecir cuáles eran los mapas climatológicos "tipo" del mundo. Así podíamos diferenciar zonas tropicales, zonas templadas y zonas frías dependiendo del lugar y los comportamientos climáticos más o menos homogéneos de esas zonas. De este modo sabemos que en los climas cálidos de la zona ecuatorial las lluvias se presentan durante todo el año alrededor de un $70 \% \mathrm{u} 80 \%$; en los de la zona tropical las lluvias aparecen, en el hemisferio norte, entre junio y septiembre (con los llamados monzones) y en el hemisferio sur, entre diciembre y marzo; y de la misma manera, en los climas cálidos desérticos las lluvias son esporádicas. Esto es un ejemplo de las tradiciones climáticas típicas que hasta ahora llegamos a discernir, en el caso anterior, con respecto a climas cálidos. Si acercamos el ejemplo a lo que más conocemos, que son nuestros climas templados podemos encontrar, por poner el caso más familiar, el del clima mediterráneo, caracterizado hasta ahora por una estación de lluvias en invierno y algunas precipitaciones en forma de lluvia en primavera y otoño, una estación seca en verano y temperaturas medias de unos 10 grados centígrados en invierno y unos 25-30 en verano (Albert, 2004: 833).

Sin embargo, por el impacto del cambio climático, los mapas están sufriendo significativas transformaciones, y con ellas se están también empezando a producir cambios en las pautas de comportamiento de las personas y modificaciones en los destinos turísticos. Ya hemos dicho en los puntos anteriores que el clima modela entornos, que las construcciones e infraestructuras de una zona se realizan en dependencia del clima habitual dado, que los consumidores turísticos consideran de manera importante el factor clima como elemento fundamental para elegir los destinos, entre otras cuestiones. Como el clima está cambiando todos los puntos que hemos analizado también se tendrán que adaptar a la nueva realidad. Estamos, pues, ante una situación sin precedentes para el turismo, pues desde que nació el turismo de masas allá por la década de los 50 del siglo XX los flujos turísticos han variado poco por motivos de clima: los países del norte han ido buscando, por regla general, zonas más cálidas. La subida de temperatura va a modificar estas pautas 
habituales de comportamiento. Se hace, por esto, necesario realizar un análisis de las repercusiones que para el turismo tendrá el cambio climático.

\section{CAMBIO CLIMÁTICO vs CAMBIO TURÍSTICO}

Es el momento de abordar las consecuencias que para el turismo pueden tener las transformaciones globales de un recurso natural tan importante como es el clima. La metamorfosis que se está produciendo es de tal alcance para todo el sistema turístico que diferentes organismos internacionales debaten ya, desde hace algún tiempo, los efectos que va a producir el cambio climático sobre el turismo. De esta manera se han configurado diversos convenios y se han realizado diferentes declaraciones a favor de un turismo sostenible que mitigue los efectos del cambio. A este respecto han surgido eventos, con mayor o menor éxito, como la Declaración de Djerba en 2003, la Declaración de Davos en 2007 o la más reciente Conferencia de Copenhague en diciembre de 2009, entre muchas otras, que se realizan para tomar conciencia de la situación a la que se ha llegado y para intentar llegar a acuerdos que vinculen a los gobiernos, diferentes agentes sociales y privados, sector turístico y demás organismos internacionales en materia de sostenibilidad para así disminuir, en la medida de lo posible, los efectos inmediatos del cambio.

Uno de esos efectos es el del llamado "calentamiento global" del planeta. El incremento progresivo de las temperaturas está siendo uno de los factores que mayores repercusiones está teniendo para que se produzca un cambio en el sistema turístico. Esta subida generalizada de temperaturas es la que "multiplica los fenómenos climáticos extremos (inundaciones y sequías, olas de calor y de frío)" (VV.AA, 2005a: 3). Vamos a ver, pues, qué efectos directos tiene este incremento de temperatura en los diferentes elementos del sistema turístico.

\section{Efectos del cambio climático sobre los destinos}

En los últimos cien años se ha comprobado que la temperatura media del planeta ha aumentado $0,6^{\circ}$ centígrados lo cual supone un incremento brusco de la temperatura. $\mathrm{Si}$ continuara esta progresión sólo harían falta 1000 años para que la temperatura media del planeta subiese, en este caso, $6^{\circ}$ centígrados, lo cual significaría que alcanzaríamos la misma variación de temperatura que naturalmente el planeta ha ido generando a lo largo de 100.000 años para pasar de una era glaciar a otra interglaciar posterior. El problema por tanto no es en sí el cambio climático sino lo apresurado del fenómeno, lo cual impide la fácil adaptación de los seres vivos a un nuevo modelo climático. Este fenómeno tiene grandes repercusiones en la geografía planetaria que afecta a la conformación de los destinos turísticos tal y como hoy se plantean. De esta manera las alteraciones más relevantes serán las siguientes:

Los destinos cuyo potencial turístico son el sol y playa tenderán a desaparecer. El importante incremento de las temperaturas hará insoportable o desagradable la estancia en algunos lugares costeros. El excesivo calor hará también que la temperatura media del mar se incremente creando situaciones de inestabilidad como la aparición de permanentes bancos de medusas y otras especies que van en detrimento de un turismo de calidad, confort y seguridad. Se producirán cambios en los ecosistemas que generarán entornos más áridos cuanto más meridional sea la zona turística, lo cual incide negativamente en los paisajes que serán poco o nada atractivos. En estas zonas más sureñas uno de los recursos fundamentales 
para la vida, el agua, será bastión para diferentes grupos de interés, lo cual implicará la aparición de graves conflictos, y de hecho podemos comprobar que "ya se está dando vigilancia militar en algunas de las cuencas más importantes del planeta para velar por las mismas, y la invasión territorial interestatal irá progresando buscando reservorios de agua potable" (Pérez, 2007: 408). Del mismo modo ya sabemos que el incremento de las temperaturas está provocando el deshielo de los casquetes polares lo que conlleva una subida del nivel del mar y los océanos que invaden las costas a razón de unos 8 ó 9 centímetros por año. Por ello diferentes asentamientos turísticos costeros que hoy conocemos pasarán a formar parte de la historia. En febrero de 2010 se publicó un artículo poniendo de manifiesto el listado de paraísos que están en peligro de extinción a causa del cambio climático y que ha sido confeccionado por diversas organizaciones ecologistas: entre esos paraísos se citan algunos como las islas Maldivas que tienen todas las papeletas para ser engullidas por el mar en el próximo siglo, de la misma manera que los humedales de Kakadú en Australia (Lenore, 2010).

Por otro lado, los destinos que están basados en un turismo de invierno y nieve tendrán que adaptarse a las nuevas circunstancias traídas por los impactos negativos de la modificación del clima. La falta de nieve y la irregularidad en las precipitaciones harán que las ocupaciones de dichas zonas sean residuales y que las temporadas sean muy limitadas en el tiempo. Las adaptaciones que se están dando ya en las zonas más al norte de Europa nos acercan claramente al problema: en algunas montañas de Noruega están creciendo árboles donde antes no se encontraba nada y la falta de nieve está provocando que se tengan que utilizar, de manera más frecuente, cañones que desperdiguen nieve artificial para que las pistas de esquí puedan ser utilizadas por los turistas. Como muestra reciente tenemos la enorme preocupación que hubo hace unos meses en Vancouver (Canadá) porque no nevaba, lo cual ponía en riesgo el éxito de los Juegos Olímpicos de invierno que se tenían que celebrar allí. Hubo que emplear una gran cantidad de cañones de nieve y transportar este elemento en camiones hacia los lugares destinados a la competición.

Así pues, los destinos más vulnerables para el turismo serán los que estén acogidos a los recursos de sol y nieve. Por su parte, los destinos urbanos, el turismo cultural, de congresos y de naturaleza será el tipo de turismo menos débil y el que se adapte mejor a los efectos del cambio climático pues sus incidencias no son tan extremas como en las otras alternativas.

\section{Efectos del cambio climático sobre los turistas}

Por lo que respecta a la demanda, ya hemos dicho que por regla general, ésta tenderá a disminuir y a cambiar de destinos, pues ya hemos advertido que el clima es un elemento motivacional de primer orden a la hora de elegir qué se quiere hacer, cómo y dónde se van a desarrollar las diferentes actividades turísticas. Se está empezando a predecir una inversión en los flujos de turistas de unos lugares a otros: hasta ahora el turismo de las zonas frías, por ejemplo del norte de Europa, buscaba zonas más cálidas en donde acomodarse. Por otro lado, los turistas de las zonas con más temperatura solían disfrutar de lo que sus zonas ya le ofrecían. La tendencia se está invirtiendo debido al calentamiento global: los turistas de las zonas más septentrionales están empezando a moverse por sus entornos que ya no son tan extremadamente fríos pero al mismo tiempo no son tan insoportablemente bochornosos como los de las zonas, por ejemplo, del Mediterráneo. Por su parte, las personas que decidían disfrutar del sol y playa en las zonas 
tradicionales, como por ejemplo el litoral Mediterráneo y sus islas, tienden a buscar otras playas situadas más al norte que les ofrezcan similares sensaciones que las que obtenían en el sur. De esta manera el consumidor turístico tenderá a trasladarse a zonas que históricamente eran más frías y así, "los turistas mediterráneos veranearán en las playas del sur de Inglaterra o similares huyendo de las insoportables temperaturas que se alcanzarán en los lugares de veraneo clásicos del sur de Europa" (Díaz, 2007). Por lo tanto los turistas del norte de Europa harán cada vez más turismo doméstico y sus visitas a los países del Mediterráneo serán menos frecuentes y menos duraderas.

Otro aspecto a tener muy en cuenta es el de los problemas de salud que acarreará el cambio climático a los turistas y que incidirán también en su toma de decisiones. Por un lado el incremento de temperaturas generará nuevas enfermedades antes no conocidas en las zonas más templadas del mundo y que suelen atribuirse a ámbitos más tropicales. La aparición de nuevos insectos como el mosquito tigre está ya haciendo estragos en algunas zonas del litoral mediterráneo, concretamente en algunas costas catalanas y andaluzas. Por otro lado se incrementarán los problemas relacionados con el sol: los casos de melanomas se han triplicado en los últimos 20 años y se espera su incremento en las próximas décadas, sobre todo porque aún no han calado las campañas preventivas debido a una ilusoria sensación de seguridad que nos confiere la falsa creencia de que con las cremas de protección solar se evita todo tipo de riesgo de sufrir un cáncer de piel (Díaz, 2007). Posiblemente, cuando las personas se conciencien de los efectos negativos que tiene darse baños de sol desproporcionados, lo cual está previsto para dentro de unos 30 años (que es el tiempo estimado para que las campañas preventivas se consoliden y actúen con fuerza en las conciencias de la gente), el valor social que hasta ahora se le da al bronceado vuelva a caer en desuso como símbolo de salud, belleza e incluso status.

\section{Efectos del cambio climático sobre los transportes}

Según algunos estudios el sector del turismo es el responsable del 5\% de las emisiones mundiales de dióxido de carbono al medio ambiente. De ese porcentaje, el 75\% procede del transporte y por lo tanto es el mayor culpable del sector en su incidencia sobre el indeseable efecto invernadero. Dentro del sector transportes es el aéreo el que tiene mayores repercusiones negativas sobre el calentamiento global del planeta: según la OMT "los viajes aéreos representan cerca del $40 \%$ de las emisiones del sector; los de carretera el 32\%, y otras formas de transporte el 3\%. El alojamiento causa alrededor del $21 \%$ del total de emisiones del sector turístico" (Olivera, 2008). Por todas estas razones el transporte no tiene más remedio que sufrir una importante y crucial renovación para mitigar los efectos nocivos sobre el medio ambiente. El advenimiento de las compañías aéreas de bajo coste supuso equilibrar la competencia con otros medios de transporte, lo que conllevó a multiplicar fuertemente su uso. Este hecho potenció el avance en la construcción de nuevas rutas y el incremento de pasajeros. Ante esta situación surge la necesidad de establecer acciones correctivas sobre el transporte en general y sobre el aéreo en particular para reducir los impactos ambientales. Por ello es bastante posible que con el tiempo se vayan estableciendo distintas normativas que limiten al máximo el uso de este tipo de transporte en tanto en cuanto la industria aérea no desarrolle tecnologías más eficientes y menos contaminantes que las actuales. Ya podemos comprobar cómo se está avanzando en una tecnología aérea "verde". De hecho desde el año 2004 se trabaja en el proyecto de un avión de propulsión solar llamado Solar Impulse que actualmente es realidad, pues ha realizado varias pruebas exitosas y la última de ellas ha sido muy reciente y novedosa, al efectuarse el primer vuelo nocturno de la historia este 2010 con 
un avión solar cuya duración fue de unas 25 horas. Del mismo modo se está intentando diseñar un zeppelín solar como medio de transporte de mercancías, aunque es sólo un proyecto virtual en la actualidad. Mientras se progresa y perfecciona la tecnología sostenible también es posible que se tome como medida adicional a las limitaciones del transporte aéreo la actuación sobre los costes del transporte de manera que las tarifas se podrían ver incrementadas y los destinos más lejanos podrían ser perjudicados por la eliminación o reducción de rutas aéreas. A este respecto "países de Asia Sudoriental, Australia, Nueva Zelanda y el Caribe, se han mostrado muy preocupados por la posibilidad de que las políticas de mitigación del calentamiento global incidan sobre su economía nacional" (Prades y De Cózar, 2008). También es posible que se fomente el uso de transportes alternativos no convencionales y ecológicos para trayectos urbanos como la bicicleta o el novedoso segway (transporte personal con autobalance). Las tecnologías ya están en marcha en la construcción de nuevos aparatos que sustituyan el consumo de los combustibles fósiles, responsables directos de la contaminación, por otros que provengan de energías renovables más limpias con el entorno (vehículos solares, etc.).

\section{Efectos del cambio climático sobre las Agencias de Viaje (AA.VV)}

La incertidumbre que adquiere esta nueva situación hace que las AA.VV varíen algunas pautas en sus modelos de gestión. Por un lado, "la inseguridad que producirán los repentinos cambios del tiempo alterará el período de reservas y la contratación del viaje, aumentará la reserva de última hora que garantizan unas mejores y más estables condiciones meteorológicas. Éste cambio en las reservas obligará a las empresas a trabajar con unos mayores niveles de flexibilidad, especialmente en el ámbito financiero, para ajustarse a las variaciones de la demanda" (VV.AA, 2005a: 38). Las AA.VV. receptivas son las que van a experimentar, de mayor manera, los efectos negativos del cambio climático pues el turismo internacional tiende a eliminar trayectos y si los hace los reduce en el tiempo. Por la misma causa los ingresos de éstas empresas turísticas se van a ver mermados, pues los flujos de turistas tienden a descender de manera significativa y los productos adquiridos por la demanda van a ser menores.

Por otro lado las AA.VV. tendrán que modificar sus modelos de actuación en tanto que deberán desplazar los destinos turísticos hacia las nuevas zonas de susceptibilidad y tendrán que innovar y reemplazar productos y servicios que antes quizá no tuvieran cabida. A este respecto ya tenemos noticias de que las empresas turísticas de las zonas del norte han iniciado ya su proceso de adaptación de una forma más evidente que las de las zonas del sur, probablemente porque los efectos del cambio climático se hacen notar mucho más en aquellas tierras que en éstas. El incremento del nivel del mar por ejemplo no es perceptible a simple vista pero el desplome de grandes bloques de hielo o la aparición de vegetación donde antes sólo había nieve nos impacta más delante de nuestros ojos. En un artículo publicado por Prades y De Cózar (2008) se nos dice que a una persona que gestiona una agencia de viajes en Qassiarsuk (al sur de Groenlandia) se le ha abierto un mundo de posibilidades con los efectos del cambio climático. Este hecho ha provocado que se generen importantes segmentos de turistas que quieren comprobar in situ los estragos causados por el calentamiento general del mundo. Es la otra cara de la moneda de las transformaciones del clima, que son nefastas para los negocios que se establecen en las zonas más cálidas y ofrecen oportunidades para las empresas turísticas que antes poco tenían que ofrecer. En plena Groenlandia está eclosionando una flora que nos deja perplejos. Bosques de coníferas 
emergen de amplios horizontes nevados. Los empresarios turísticos de esa zona deducen que la gente quiere asistir a estos lugares porque intuyen que dejarán de existir tal y como actualmente se dan en no mucho tiempo. Además el clima permite que puedan realizarse estos viajes que antes a nadie se le hubiera pasado por la cabeza por lo inhóspito de la zona. De la misma manera "mentes similares han organizado excursiones al glaciar Perito Moreno, en Argentina, para observar cómo caen los casquetes sobre el océano o cómo los fiordos noruegos desaparecen poco a poco. En Finlandia la nieve derretida ha despejado caminos donde antes sólo había hielo y las principales agencias de viajes finlandesas han empezado a sustituir las motonieve por los autocares. Están satisfechos por la pujanza de sus negocios: los inviernos más suaves y cortos incrementan el turismo" (Prades y De Cózar, 2008). Así el cambio climático hará que las agencias de viajes varíen sus formas de trabajar en función de la zona del mundo donde estén instaladas: se le abrirán nuevas posibilidades a las que estén en latitudes más altas y serán más vulnerables las que se asienten en zonas más cálidas.

\section{CONCLUSIONES}

A lo largo de estas páginas hemos podido acercarnos a un problema crucial con el que se enfrenta el turismo en las próximas décadas. El elemento destino es el eslabón llave del sistema turístico y dentro de él, sobre todo, el recurso natural que implica una gran cantidad de factores como los paisajes, la flora, la fauna, los elementos hídricos, el clima y tantos otros. Y decimos que es un eslabón llave porque sus acciones vienen determinadas en gran parte por los caprichos de la naturaleza que en muchas ocasiones son incontrolables frente al resto de los elementos del sistema. Un cambio como el que se está produciendo en el clima a nivel global tiene unas repercusiones tan extensas en el conjunto del turismo que no podemos negarle importancia.

Las transformaciones ya se están produciendo, pero lo único que podemos hacer es realizar proyecciones de futuro si las situaciones continúan con la misma inercia. Las variaciones en las acciones más sostenibles o nada sostenibles que se hagan en el futuro nos permitirán seguir la pauta de la gran transformación del turismo o su regreso a las condiciones todavía cotidianas. Al final lo que nos encontramos es un panorama en donde se pierde diversidad en cuanto que las posibilidades de acceder a zonas más frías tiende a desaparecer tal y como hemos visto. El mundo del turismo se adaptará a todas las circunstancias que se vayan presentando. Lo asumirá de mejor o de peor talante pero no dejará de desarrollarse. El que definitivamente pierde es el ser humano porque ve limitadas sus posibilidades, la multiplicidad de experiencias y de sensaciones que todos los aspectos de la naturaleza le pueden otorgar y que no podrán nunca ser reemplazados por lo artificial.

El turismo probablemente sea como la energía, que ni se crea ni se destruye, sólo se transforma, y eso es lo que está aconteciendo en la actualidad: nuevas pautas climáticas, nuevos mercados, consumidores turísticos, motivaciones, tecnología, etc. En definitiva, los nuevos marcos de referencia del turismo están en estrecha ligazón con los problemas del cambio climático cuyas consecuencias fundamentales serán la vulnerabilidad de las zonas (unas más que otras), y la adaptación de la oferta y la demanda turística en cuanto a aspectos como el comportamiento, la sensibilización con cuestiones medioambientales, la brevedad de las estancias, la desviación hacia otras zonas turísticas y la desestacionalidad del turismo. 


\section{BIBLIOGRAFÍA}

ALBERT PIÑOLE, I. (2004): Gestión, productos y servicios de las agencias de viajes, Madrid, Centro de Estudios Ramón Areces.

- (2008): "Globalización y turismo", en Uña, O.; Hormigos, J. y Martín, A: Las dimensiones sociales de la globalización, Madrid, Paraninfo, pp. 185-228.

DÍAZ CANO, M. y DÍAZ CANO, E. (2009): "El lado oscuro de la sociedad actual: los residuos", en Barataria. Revista Castellano-Manchega de Sociología, Toledo, ACMS, n ${ }^{\circ}$ 10, pp. 123-140.

DÍAZ PRIETO, M. (2007): "Turismo de lluvia", disponible en http://hemeroteca.lavanguardia.es/pre view/ 1963/04/07/pagina12/60059171/pdf.html?search=turismo\%20de\%201luvia\%20diaz\%20prieto

GÓMEZ MARTíN, B. (1999): "La relación clima-turismo: consideraciones básicas en los fundamentos teóricos y prácticos". Investigaciones Geográficas, nº 21, pp. 21-34.

LENORE, V. (2010): "Paraísos que el cambio climático podría destruir", disponible en http://es.viajes.yahoo.com/actualidad/lugares_en_peligro_cambio_climatico.html

OLIVERA BONILLA, A. (2008): Del cambio climático al desastre turístico, México Greenpeace.

PÉREZ REDONDO, R. J. (2007): "El poder del agua en las sociedades: un análisis de la situación actual”, en: Pérez, R. J. y Martín, A. (coords.): Castilla-La Mancha: 25 años de autonomía. XII Congreso Nacional de Sociología en Castilla-La Mancha, Toledo, ACMS, pp. 403-410.

- (2008): "El medio ambiente ante una sociedad globalizada", en Uña, O.; Hormigos, J. y Martín, A: Las dimensiones sociales de la globalización. Madrid, Paraninfo, pp. 115-132.

PRADES, J. y DE CÓZAR, A. (2008): "Cambio climático, cambia el turismo”, disponible en http://www.elpais.com/articulo/sociedad/Cambio/climatico/cambia/turismo/elpepisoc.html.

URTEAGA, E. (2008): "La sociedad civil en cuestión", en Barataria. Revista Castellano-Manchega de Sociología, Toledo, ACMS, $\mathrm{n}^{\circ}$ 9, pp. 155-188.

VV.AA. (2005a): El cambio climático. Efectos sobre el turismo, Madrid, ISTAS.

VV.AA. (2005b): Sociedad del conocimiento, Barcelona, UOC.

\section{Breve currículo:}

Licenciado en Sociología por la Universidad Pontificia de Salamanca. Cuenta con experiencia docente e investigadora en la Universidad de Castilla-La Mancha. En la actualidad es profesor de la Universidad Rey Juan Carlos impartiendo las asignaturas Sociología del Turismo y del Ocio y Turoperadores y Agencias de Viajes, entre otras, e investigador de la Cátedra de Turismo de Madrid. Ha participado en diversas publicaciones y diferentes Congresos nacionales. 